J. Clin. Chem. Clin. Biochem.

Vol. 21, 1983, pp. 279-286

\title{
Diagnostische Wertigkeit der Lysozymaktivität bei neurologischen Erkrankungen in Liquor und Serum
}

\author{
Von $U$. Kauerz und B. Weisner \\ Neurologische Universitäts-Kernklinik mit Poliklinik (Direktor Prof. Dr. K. Kunze) der Universität Hamburg
}

(Eingegangen am 19. Januar/1. Dezember 1982)

Zusammenfassung: Die Lysozymaktivität wurde an Hand der Lyse von Bakterienzellwänden bei Micrococcus lysodeicticus mit der Lysoplate-Methode und mit der Turbidimetrie bestimmt. Beide Methoden wurden miteinander verglichen.

Die Lysozymaktivität in Liquor und Serum wurde bei Referenzpersonen $(n=57)$ und bei Patienten mit bakteriellen Meningoencephalitiden $(n=20)$, akuten viralen Meningoencephalitiden $(n=29)$, multipler Sklerose $(n=19)$, chronischen Entzündungen $(n=29)$, Subarachnoidalblutungen $(n=4)$, Diskusprotrusionen $(n=12)$, Polyneuropathien $(n=8)$, Neoplasmen $(n=28)$, cerebralen Gefäßprozessen $(n=2)$, SchädelHirn-Traumen $(n=3)$, metabolischen Myelopathien $(n=7)$, Hydrocephali $(n=2)$ bestimmt.

Referenzpersonen überschritten im Liquor eine Lysozymaktivitätskonzentration von 27000 Lysozymeinheiten pro Liter nicht.

Katalytische Aktivitätskonzentrationen über 105000 Lysożymeinheiten wurden besonders häufig bei bakteriellen Meningoencephalitiden beobachtet, aber auch bei anderen nicht bakteriell entzündlichen Erkrankungen des Zentralnervensystems gefunden. Die Lysozymaktivität im Liquor zeigte keine Korrelation mit den Liquor-Kenngrößen Zellzahl, Albumin. Auch bestand kein Zusammenhang mit der katalytischen Aktivität im Serum. Nur bei bakteriellen Meningoencephalitiden konnte ein statistisch signifikanter Zusammenhang nachgewiesen werden fựr diè Zunahme der Lysozymaktivität im Liquor bei Abnahme der Glucosekonzentration.

Die Befunde sprechen für einen Einstrom von Lysozym aus den liquornahen Geweben, in denen es zur Anreicherung von Granulocyteñ ünd Makrophagen kommen kann. Die Lysozymaktivität erwies sich als besonders empfindliche Verlaufs-Kenngröße während der Behandlung einer bakteriellen Meningoencephalititis. Die Lysozymaktivität im Liquor fạ̈lı̣t mit steigender Anțibiotikakonzentration im Liquor ab.

Diagnostic Validity of Lysozyme Catalytic Activity in Cerebrospinal Fluid and Serum in Neurologic Diseases

Summary: Lysozyme catalytic activity was determined by lysis of cell-walls of Micrococcus lysodeicticus, using the Lysoplate method and turbidimetry. The two methods were compared.

Lysozyme catalytic activity was determined in cerebrospinal fluid and serum of reference persons $(n=57)$ and patients with bacterial meningitis $(n=20)$, viral meningoencephalitis $(n=29)$, multiple sclerosis $(n=$ 19), chronic inflammatory diseases $(n=29)$, subarachnoid haemorrhage $(n=4)$, prolaps of the intervertebral disk $(n=12)$, polyneuropathy $(n=8)$, neoplasm $(n=28)$, cerebral angiopathy $(n=2)$, cerebral trauma $(n=$ $3)$, metabolic myelopathy $(n=7)$, hydrocephalus $(n=2)$.

In reference persons lysozyme catalytic activity concentration did not exceed 27000 lysozyme units per liter. Catalytic activity concentration higher than 105000 lysozyme units per liter was very often found in CSF of cases with bacterial meningitis and also in CSF of patients with other, nonbacterial inflammatory diseases of the CNS. 
Lysozyme catalytic activity in CSF showed no correlation with cell count and albumin concentration in CSF. There was also no correlation with the lysozyme catalytic activity in serum. Only the CSF of patients with bacterial meningoencephalitis showed a statistically significant correlation between the increase of lysozyme catalytic activity in CSF and the decrease of glucose concentration.

These findings indicate an influx of lysozyme from tissues surrounding the CSF, where granulocytes and macrophages may be enriched.

Lysozyme catalytic activity was a very sensitive parameter during the course of a bacterial meningitis. The enzyme catalytic activity in CSF decreases with increasing concentration of antibiotics in the CSF.

\section{Einführung}

Eine erhöhte Lysozymaktivität im Liquor wurde bei Patienten mit bakteriellen Meningitiden gefunden.

Aber auch andere neurologische Erkrankungen können mit einer erhöhten Lysozymaktivität einhergehen. Bisher ist nicht geklärt, ob dieses Enzym weitgehend von Strukturen des liquornahen Raumes stammt oder ausschließlich aus dem Plasma in den Liquor übertritt. In Liquor und Serum von Referenzpersonen und Patienten mit neurologischen Erkrankungen wurde die Lysozymaktivität gemessen und untersucht auf:

1. die Abhängigkeit zwischen den Kompartimenten Liquor/Serum,

2. die Abhängigkeit zur Albuminkonzentration im Liquor als einer Kenngröße zur Beurteilung der gestörten Blut-Liquor-Schrankenfunktion,

3. den Zusammenhang mit der Zellzahl und der Glucosekonzentration im Liquor.

Die diagnostische Aussagefähigkeit der Lysozymaktivität im Liquor wurde an größeren Patientenkollektiven neurologischer Erkrankungen überprüft.

\section{Material und Methoden}

Durch Lumbalpunktion gewonnene Liquores, eingesandt im Zeitraum von 1980 bis 1981 in das Liquorlabor der Neurologischen Universitätsklinik, wurden in die Untersuchung einbezogen.

Unmittelbar nach der Entnahme der Probe erfolgte die Bestimmung der Zellzahl, die Zentrifugation und die Lagerung bei $+4^{\circ} \mathrm{C}$ in Polyethylengefäßen (Fa. Eppendorf 3810).

Blut wurde abzentrifugiert und das Serum ebenso behandelt und aufgearbeitet. Spätestens am dritten Tag erfolgte die Bestimmung der Lysozymaktivität.

\section{Referenzpersonen}

Als Referenzpersonen $(n=57)$ dienten solche Probandeñ, die zum Ausschluß einer neurologischen Erkrankung punktiert worden waren, der Verdacht sich jedoch nicht erhärten ließß. In diesen Liquorproben betrug die Zellzahl bis 4 Leukocyten pro $\mu \mathrm{l}$ (FuchsRosenthal-Zählkammer), die Trübung in der Normomastixreạktion bis Grad V, Gesamteiweiß bis $520 \mathrm{mg} / \mathrm{l}$, Albumin bis 320 $\mathrm{mg} / \mathrm{l}$.

\section{Patienten}

Die Patienten wurden nach klinischen Diagnosen geordnet: Bakterielle Meningitiden $(n=20)$, virale Meningoencephalitiden ( $n$ $=29)$, Multiple Sklerose $(n=19)$, andere chronische Entzündungen des Zentralnervensystems $(n=29)$, und andere neurologische Syndrome (Subarachnoidalblutungen $n \equiv 4$, Discusprotrusionen $n=12$, Polyneuropathien $n=8$, Neoplasmen $n=28$, cerebrale Gefäßprozesse $n=2$, Schädel-Hirn-Traümen $n=3$, metabolische Myelopathien $n=7$, Hydróocēphạli $n=2$ ).

\section{Methoden}

Die Lysozymaktivität wurde gemessen mit Hilfe der Lyse von Bakterienzellwänden (Micrococcus lysodeicticus) durch die Muramidase Lysozym (EC 3.2.1.17). Eine Lysozymeinheit $\left(25^{\circ} \mathrm{C}\right.$; $\mathrm{pH}$ $7,0)=\Delta \mathrm{A} 0,001 / \mathrm{min}$ bei $1 \mathrm{~cm}$ Schichtdicke. Verglichen wurden ein Diffusionstest und eine turbidimetrische Methode.

\section{Beiden Methoden gemeinsame Reagentien:}

Als Substrat dienten Bakterien des Stammes Micrococcus lysodeicticus (Fa. Koch-Light Laboratories). Als Standard diente menschliches Lysozym (Fa. Selfact AB., Stockholm).

Als Präzisions- und Richtigkeitskontrolle diente eine unabhängig von den Standardlösungen hergestellte :Verdünnung der Standạrdlösung (Selfact AB., Stockholm).

\section{Diffusionstest (16)}

$0,4 \mathrm{~g}$ getrocknete Bakterien wurdèn in 15 mol eines Phosphatpuffers $\left(634 \mathrm{ml} 67 \mathrm{mmol} / 1 \mathrm{KH}_{2} \mathrm{PO}_{4}\right.$ in $150 \mathrm{mmol} / \mathrm{l} \mathrm{NaCl}+390 \mathrm{ml} 67$ $\mathrm{mmol} / \mathrm{l} \mathrm{Na} \mathrm{HPO}_{4}$ in $150 \mathrm{mmol} / \mathrm{l} \mathrm{NaCl}$ ) über Nacht mit Hilfe eines Magnetrührers bei $+4^{\circ} \mathrm{C}$ suspendiert. $1 \mathrm{~g}$ Agarose (Fa. Behringwerke, Marburg) wurde am folgenden Tag in $50 \mathrm{ml}$ dest. Wasser gelöst unter Rühren und Erwärmen auf $100^{\circ} \mathrm{C}$. Anśchließend wurde die Lösung auf $52^{\circ} \mathrm{C}$ temperiert. Zu der Agaroselösung wurden $50 \mathrm{ml}$ des ebenfalls auf $52^{\circ} \mathrm{C}$ erwärmten Phosphatpuffers ( $\mathrm{pH}=6,5$ ), versetzt mit $0,2 \mathrm{ml} 1-$ Octanol und $10 \mathrm{mg}$ Thimerosal (Koch-Light-Laboratories), hinzugegeben: Schließ̣liç wìurde auch die kurz auf $52^{\circ} \mathrm{C}$ erwärmte Micrococcus-Suspeñsión hinzzúgemischt. Jeweils $12 \mathrm{ml}$ dieses Gemisches wurden in Pẹtrischchalen (Durchmesser etwa $11 \mathrm{~cm}$ ) ausgegössen, nach Erkalten Offnun= gen ausgestanzt (Durchmesser $4 \mathrm{~mm}$ ) und je $10 \mu \mathrm{l}$ der verdünnten Standardölösungen, Serựm und Liquor unverdünnt eingefüllt. Nach 24stündiger Inkubation bei $+37^{\circ} \mathrm{C}$ entstanden. L̀sehöfe um die Einfüllöffrnüung, die die Lyse der Bakterien durch das Ënzym anzeigten. Der Durchmesser der Lysehöfe verhielt sich proportional żu der Enzymaktivität. An Hand der Verdünnungen der Standardlösung ließ sich eine Standardkurve herstellen (nicht linearer Verlauf) und an dieser die unbekannte Lysozymaktivität in den Proben exakt bestimmen. Die Kalibrierung erfolgte in Lysozymeinheiten. Die untere Nachweisbarkeitsgrenze lag bei 10000 Lysozymeinheiten pro Liter. $\therefore$ 


\section{Turbidimetrischer Test (17)}

In einer Lösung aus Phosphatpuffer $(67 \mathrm{mmol} / \mathrm{l}, \mathrm{pH}=6,3)$ und $\mathrm{NaCl}(15,4 \mathrm{mmol} / \mathrm{l})$ wurde Micrococcus lysodeicticus $(0,2 \mathrm{~g} / \mathrm{l}) \mathrm{su}-$ spendiert und nach 10 Minuten Temperierung auf $+25^{\circ} \mathrm{C}$ zur Bestimmung eingesetzt (17). Verwandt wurden Testomar-Testansätze der Fa. Behringwerke, Marburg. $1,5 \mathrm{ml}$ dieser Bakteriensuspension wurden mit $0,025 \mathrm{ml}$ Standardlösung $(1: 120=$ 668300 Lysozymeinheiten pro Liter bis $1: 1920=41700$ Lysozymeinheiten pro Liter $=$ geometrische Verdünnungsreihe) oder Liquor oder Serum vermischt, in jeweils eine auf $+25^{\circ} \mathrm{C}$ vortemperierte Halbmikroküvette $(1 \mathrm{~cm}$ Schichtdicke) gegossen und beginnend nach 30 Sekunden -4 Absorptionen, entsprechend 3 Absorptionsdifferenzen im Abstand von 1,5 Minuten gemessen (Wellenlänge $546 \mathrm{~nm}$, Spektrallinienphotometer Eppendorf, Typ $1101 \mathrm{M})$. Der Mittelwert der Absorptionsdifferenzen wurde ermittelt und - bei den Standardlösungen - gegen die bekannte Enzymaktivität im Ḱoordinatenkreuz aufgetragen. Die so hergestellte Standardkurve hatte einen nicht-linearen Verlauf. Der Mittelwert der Absorptionsdifferenzen unbekannter Proben wurde ebenfalls berechnet und die unbekannte Enzymaktivität an Hand der vorher erstellten Standardkurve abgelesen.

War die mittlere Absorptionsdifferenz größer als 0,1, so wurde die Probe 1:4 mit physiologischer $\mathrm{NaCl}$-Lösung verdünnt.

Die Zellzählung erfolgte mit der Zählkammer nach Fuchs-Rosenthal(Leukocytenkonzentrationsangabe in $\mathrm{n} / \mu \mathrm{l}$ ).

Die Glucose wurde in Liquor und Serum bestimmt mit der Glucoseoxidase-Perid-Methode (Präzision von Tag zu Tag $=4 \%$, Richtigkeit von $\mathrm{Tag} z \mathrm{Tag}=5 \%, \mathrm{n}=21$ ). Albumin und $\mathrm{IgG}$ wurden quantitativ bestimmt mit der kinetisch immunnephelometrischen Methode (ICS-System, Beckman, Fullerton, Californien - Richtigkeit von Tag zu Tag: Albumin $+3 \%$, IgG $+3 \%$, (21)).

Statistische Auswertungen

Die Verteilung der Meßwerte wurde im linearen und logarithmischen Wahrscheinlichkeitsnetz überprüft. Um den stochastischen Zusammenhang der klinisch-chemischen Daten zu erkennen, wurde der bivariate Korrelationskoeffizient (Pearson-r) errechnet und auf Signifikanz gegen Null geprüft (18).

\section{Ergebnisse}

Zum Vergleich des Diffusionstests und der turbidimetrischen Methode wurden 36 Seren von Patienten nativ eingesetzt. Die Verteilung der Wertepaare gibt Abbildung 1 wieder. Gleichzeitig eingetragen ist die bivariate Regressionsgerade $(y \equiv 0,824 x-13,49)$. Der Regressionskoeffizient (Pearson-r) beträgt 0,95 , signifikant auf dem $0,1 \%$-Niveau.

Die Qualitätskontrolle in der Serie ergab einen VK von $0,39 \%$ und eine Abweichung vom Sollwert von $+1,82 \%(n=10)$ für den Diffusionstest. Für den turbidimetrischen Test lag der VK bei 3,8\% und die Abweichung vom Sollweit bei $+13,12 \%(n=10)$.

Die Qualitätskontrolle von Tag zu Tag ergab für den Diffusionstest $(n=19)$ einen VK von $9,06 \%$, die Abweichung vom Sollwert betrug $+9,37 \%$.

Die untere quantitative Nachweisbarkeitsgrenze lag bei beiden Methoden bei 10000 Lysozymeinheiten . pro Liter. Erkennbare Diffusionsringe unterhalb eines Durchmessers von $4,5 \mathrm{~mm}$ konnten jedoch nicht mehr quantitativ ausgewertet werden.

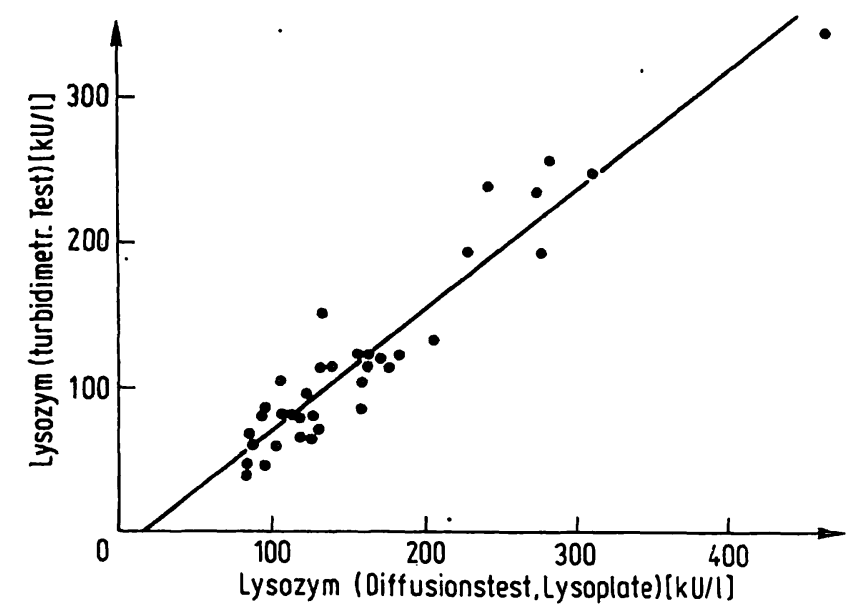

Abb. 1. Wertepaare der Lysozymaktivität gemessen mit dem Lysoplate-Test (Abszisse) und mit der turbidimetrischen Methode (Ordinate). Eingetragen ist die bivariate $\mathrm{Re}-$ gressionsgerade ( $y=0,824 x-13,49)$. Der Korrelationskoeffizient (Pearson-r) beträgt 0,954 (signifikant auf dem $0,1 \%$-Niveau).

Für die Bestimmungen bei Referenzpersonen und Patienten wurde der Diffusionstest eingesetzt.

Haltbarkeit der Probe

Úberprüft an Kontrollen und 5 unbekannten Serumproben ergab sich kein Aktivitätsverlust des Enzyms bei $-20^{\circ} \mathrm{C}$ bis zum 21 . Tag nach Entnahme und kein Aktivitätsverlust bei $+4^{\circ} \mathrm{C}$ bis zum 3 . Tag nach der Probenentnahme.

\section{Klinisch-chemische Befunde}

Abbildung 2 gibt die 95\% Vertrauensbereiche von Referenzpersonen und von Patienten mit neurologi-

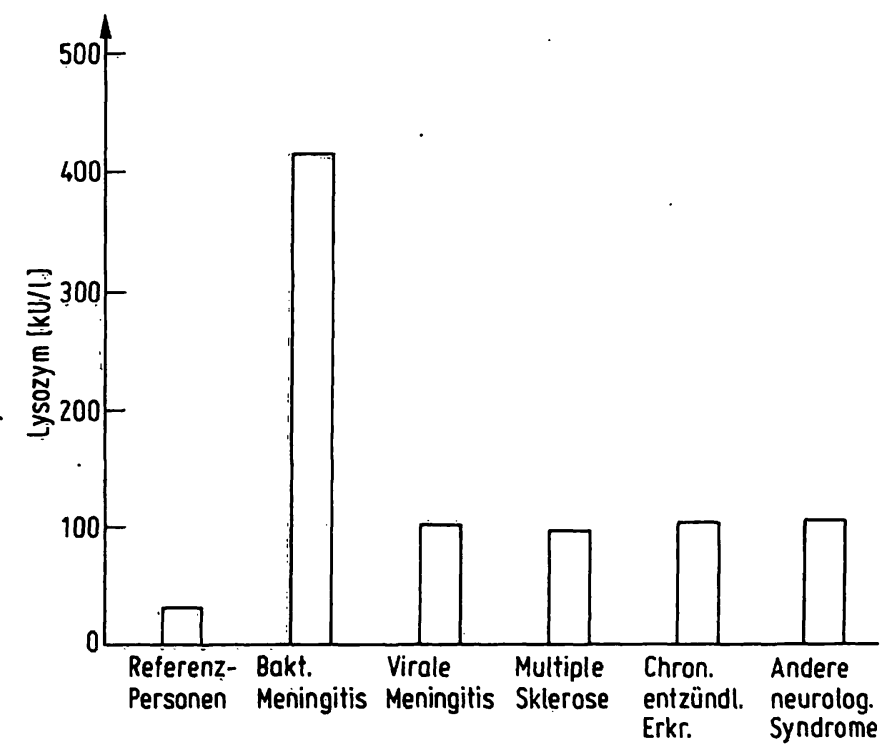

Abb. 2. $95 \%$-Vertrauensbereich der Lysozymaktivität im lumbalen Liquor. Referenzpersonen $n=57$, Patienten $n=$ 163. Andere neurologische Syndrome: Subarachnoidalblutungen $(n=4)$, Disçusprotrusionen $(n=12)$, metabolische Polyneuropathien $(n=8)$, Neoplasmen $(n$ $=28)$, cerebrale Gefäßprozesse $(n=2)$, Schädel-, HirnTraumen $(n=3)$, Myelopathien $(n=7)$, Hydrocephalus $(n=2)$. 
schen Erkrankungen wieder. Der Referenzbereich reichte bis 27000 Lysozymeinheiten pro Liter. Dieser Referenzbereich wurde bei allen untersuchten Krankheitsgruppen überschritten (Abb. 2). Die Bestimmung der Lysozymaktivität ermöglichte keine Diskriminierung zwischen den einzẹlnen Krankheitsgruppen.

Durchschnittlich besonders hoch fiel die Lysozymaktivität bei bakteriellen Meningitiden aus. Werte über 105000 Lysozymeinheiten pro Liter wurden jedoch nicht nur bei bakteriellen Meningitiden, sondern auch bei anderen Erkrankungen des Zentralnervensystems entzündlicher und nicht entzündlicher Genese erreicht (Tab. 1).

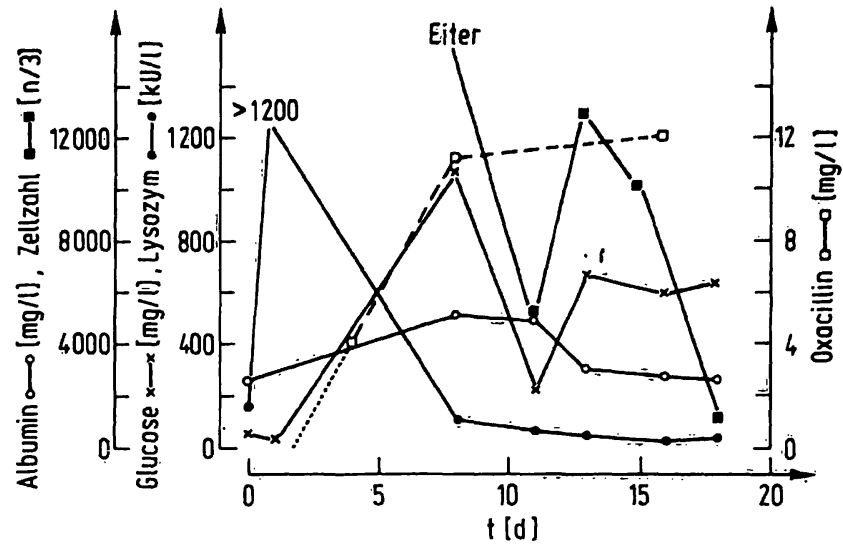

Abb. 3. Konzentrationen im Liquor im Verlauf einer bakteriellen Meningoencephalitis. K.A., 53 Jahre, o. Erreger: Staphylococcus aureus, antibiotische Therapie: Oxacillin, Gentamycin.

Tab. 1. Patienten mit hoher Lysozymaktivität in lumbal entnommenem Liquor ohne Nachweis einer bakteriellen Erkrankung.

\begin{tabular}{|c|c|c|c|c|c|c|c|c|}
\hline Name & $\begin{array}{l}\text { Alter } \\
{[\mathrm{a}]}\end{array}$ & $\because i^{\text {Ge- }}$ schlecht & $\begin{array}{l}\text { Lysozym } \\
\text { [Lysozym- } \\
\text { einheiten } \\
\text { pro Liter] }\end{array}$ & $\begin{array}{l}\text { Zellzahl } \\
{[\mathrm{n} / \mu \mathrm{l}]}\end{array}$ & $\begin{array}{l}\text { Albumin } \\
{[\mathrm{mg} / \mathrm{l}]}\end{array}$ & $\begin{array}{l}\mathrm{IgG} \\
{[\mathrm{mg} / \mathrm{l}]}\end{array}$ & $\begin{array}{l}\text { Glucose } \\
{[\text { mg/l] }}\end{array}$ & Diagnose \\
\hline K.K. & 40 & $\delta$ & 1200000 & 2 & 1068 & 122 & 688 & Wernicke-Ēncephalopathie \\
\hline J.P. & 58 & $\delta$ & 330000 & - & 232 & 33 & - & Hypophysentumor \\
\hline H.M. & 43 & $q$ & 1200000 & - & 176 & 22 & - & Hydrocephalus internus \\
\hline S.W. & 59 & $\delta$ & 1200000 & 1 & 180 & 22 & 728 & Encephalopathie (Alkohol-Abusus) \\
\hline C.G. & 49 & $\delta$ & 390000 & 1 & 327 & 68 & 523 & Cephalgien \\
\hline S.H. & 63 & $q$ & 305000 & 4 & 123 & 43 & - & Jakob-Creutzfeldt \\
\hline F.B. & 31 & q & 690000 & 3 & 158 & 25 & 588 & Encephalopathie nach Prolactinom \\
\hline S.E. & 74 & $\delta$ & 270000 & 11 & 7056 & 1584 & 448 & ungeklärt \\
\hline
\end{tabular}

Senkung der Lysozymaktivität im Verlauf einer antibiotisch behandelten bakteriellen Meningoencephalitis

Abbildung 3 zeigt den Verlauf einer bakteriellen Meningoencephalitis. $\mathrm{Zu}$ Beginn der Erkrankung war die Lysozymaktivität niedrig bei eitrigem $\mathrm{Li}$ quor. An den folgenden Tagen stieg sie sehr stark an bei gleichzeitigem Beginn einer Antibiotikatherapie. Mit ansteigender Antibiotikakonzentration im Liquor fiel die Lysozymaktivität nahezu spiegelbildlich ab als Hinweis auf einen nicht nur zeitlichen, sondern auch kausalen Zusammenhang. Im Verlauf verhielt sich die Glucosekonzentration im Liquor genau entgegengesetzt zur Lysozymaktivität. Ein zeitlicher Zusammenhang zwischen der Zellzahl im Liquor und der Lysozymaktivität ließ sich nicht erkennen.

\section{Zusammenhang zwischen Lysozymaktivität und Glu- kosekonzentration im Liquor}

In der Gruppe der bakteriellen Meningoencephalitiden fiel die Korrelation der Lysozymaktivität mit der Glucosekonzentration negativ aus. Dieses Ergebnis war hochsignifikant $(\alpha=0,1 \%)$. Es wies auf einen zunehmenden Glucoseverbrauch mit ansteigender Lysozymaktivität hin. Bei den anderen Krankheitsgruppen und bei den Referenzpersonen war ein solcher Zusammenhang nicht nachweisbar (Tab. 2).

Zusammenhang zwischen der Lysozymaktivität ünd der Zellzahl im Liquor

Nur bei Patienten mit viralen Meningoencephalitiden (signifikant $\alpha=0,1 \%$ ) und bei Patienten mit multipler Sklerose (signifikant $\alpha=1,0 \%$ ) zeigte sich eine positive Korrelation zwischen Zellzahl und Lysozymaktivität im Liquor mit hoher Signifikanz (Tab. 3).

Zusammenhang zwischen der Lysozymaktivität und der Albuminkonzentration im Liquor

Bei Referenzpersonen und Patientèn konnte kein Zusammenhang ermittelt werden zwischen der Lysozymaktivität und der Albuminkonzentration im Liquor (Tab. 4). 
Tab. 2. Glucose und Lysozymaktivität in lumbal entnommenem Liquor bei Referenzpersonen und bei Patienten mit neurologischen Syndromen. $\mathrm{n}=$ Zahl der Fälle, $\overline{\mathrm{x}}, \overline{\mathrm{y}}=$ Mittelwert, $\mathrm{s}_{\mathrm{x}, \mathrm{y}}=$ einfache Standardabweichung, $\mathrm{r}=$ Korrelationskoeffizient (Pearson-r), $\alpha=$ Irrtumswahrscheinlichkeit. Andere neurologische Syndrome $(n=51)$ : Subarachnoidalblutungen $(n=4)$, Discusprotrusionen $(n=12)$, metabolische Polyneuropathien $(n=8)$, Neoplasmen $(n=28)$, cerebrale Gefäßprozesse $(n=2)$, Schädel-HirnTraumen $(n=3)$, Myelopathien $(n=7)$, Hydrocephalus $(n=2)$.

\begin{tabular}{|c|c|c|c|c|c|c|c|c|c|}
\hline \multirow[t]{2}{*}{ Probanden } & \multirow[t]{2}{*}{$\mathbf{n}$} & \multicolumn{2}{|c|}{$\begin{array}{l}\text { Glucose im Liquor } \\
\text { [mg/l] }\end{array}$} & \multicolumn{2}{|c|}{$\begin{array}{l}\text { Lysozym im Liquor } \\
\text { [Lysozymeinheiten } \\
\text { pro Liter] }\end{array}$} & \multicolumn{3}{|c|}{ Regression } & \multirow{2}{*}{$\begin{array}{l}\text { Korrela- } \\
\text { tion } \\
\text { signifikant } \\
\qquad \alpha=0,1 \%\end{array}$} \\
\hline & & $\overline{\mathbf{x}}$ & $\mathbf{s}_{\mathbf{x}}$ & $\overline{\mathbf{y}}$ & $s_{y}$ & $\mathbf{r}$ & $a_{0}$ & $a_{1}$ & \\
\hline Referenzpersonen & 12 & 600,42 & 90,87 & 15630 & 4850 & $-0,132$ & 19,85 & $-0,01$ & nein \\
\hline Patienten & & & & & & & · & & \\
\hline $\begin{array}{l}\text { Bakt. Meningitis } \\
\text { Virale Meningitis } \\
\text { Multiple Sklerose } \\
\text { Chron. entzündl. Erkrankungen } \\
\text { Andere neurologische Syndrome }\end{array}$ & $\begin{array}{l}15 \\
13 \\
13 \\
16 \\
20\end{array}$ & $\begin{array}{l}464,40 \\
572,69 \\
574,23 \\
591,13 \\
575,25\end{array}$ & $\begin{array}{r}156,59 \\
82,66 \\
.75,85 \\
22,78 \\
90,30\end{array}$ & $\begin{array}{r}207600 \\
36150 \\
42230 \\
37380 \\
43950\end{array}$ & $\begin{array}{r}304610 \\
30200 \\
34660 \\
21730 \\
26920\end{array}$ & $\begin{array}{r}-0,875 \\
-0,457 \\
0,071 \\
0,453 \\
0,035\end{array}$ & $\begin{array}{r}997,58 \\
131,81 \\
23,63 \\
-217,98 \\
37,97\end{array}$ & $\begin{array}{r}-1,70 \\
-0,17 \\
0,03 \\
0,43 \\
0,01\end{array}$ & $\begin{array}{l}\text { ja } \\
\text { nein } \\
\text { nein } \\
\text { nein } \\
\text { nein }\end{array}$ \\
\hline
\end{tabular}

Tab. 3. Zellzahl und Lysozymaktivität in lumbal entnommenem Liquor bei Referenzpersonen und bei Patienten mit neurologischen Syndromen.

\begin{tabular}{|c|c|c|c|c|c|c|c|c|c|c|}
\hline \multirow[t]{2}{*}{ Probanden } & \multirow[t]{2}{*}{$\mathrm{n}$} & \multicolumn{2}{|c|}{$\begin{array}{l}\text { Zellzahl } \\
\text { im Liquor } \\
{[\mathrm{n} / \mu \mathrm{l}]}\end{array}$} & \multicolumn{2}{|c|}{$\begin{array}{l}\text { Lysózym im Liquor } \\
\text { [Lysozymeinheiten } \\
\text { pro Liter] }\end{array}$} & \multicolumn{3}{|c|}{ Regression } & \multicolumn{2}{|c|}{$\begin{array}{l}\text { Korrelation } \\
\text { signifikant }\end{array}$} \\
\hline & & $\overline{\mathbf{x}}$ & $s_{x}$ & $\overline{\mathbf{y}}$ & $\mathrm{sy}_{\mathrm{y}}$ & $\mathbf{r}$ & $a_{0}$ & $\mathbf{a}_{1}$ & $\begin{array}{l}\alpha= \\
0,1 \%\end{array}$ & $\begin{array}{l}\alpha= \\
1,0 \%\end{array}$ \\
\hline Referenzpersonen & 19 & 2 & 1 & 17110 & 4910 & $-0,163$ & 18,41 & $-0,51$ & nein & nein \\
\hline \multicolumn{11}{|l|}{ Patienten } \\
\hline $\begin{array}{l}\text { Bakt. Meningitis } \\
\text { Virale Meningitis } \\
\text { Multiple Sklerose } \\
\text { Chron. entzündl. Erkrankungen } \\
\text { Andere neurologische Syndrome }\end{array}$ & $\begin{array}{l}16 \\
20 \\
15 \\
29 \\
32\end{array}$ & $\begin{array}{r}632 \\
30 \\
9 \\
7 \\
2\end{array}$ & $\begin{array}{r}1259 \\
58 \\
11 \\
11 \\
3\end{array}$ & $\begin{array}{r}164310 \\
42750 \\
38800 \\
45540 \\
49720\end{array}$ & $\begin{array}{r}144790 \\
32300 \\
33340 \\
29590 \\
38060\end{array}$ & $\begin{array}{r}-0,040 \\
0,756 \\
0,643 \\
-0,050 \\
0,114\end{array}$ & $\begin{array}{r}167,19 \\
30,18 \\
21,39 \\
45,35 \\
45,94\end{array}$ & $\begin{array}{r}-0,00 \\
0,14 \\
0,65 \\
-0,04 \\
0,50\end{array}$ & $\begin{array}{l}\text { nein } \\
\text { ja } \\
\text { nein } \\
\text { nein } \\
\text { nein }\end{array}$ & $\begin{array}{l}\text { nein } \\
\mathrm{ja} \\
\mathrm{ja} \\
\text { nein } \\
\text { nein }\end{array}$ \\
\hline
\end{tabular}

Tab. 4. Albuminkonzentration und Lysozymaktivität in lumbal entnommenem Liquor bei Referenzpersonen und bei Patienten mit neurologischen Syndromen.

\begin{tabular}{|c|c|c|c|c|c|c|c|}
\hline \multirow[t]{2}{*}{ Probanden } & \multirow[t]{2}{*}{$\dot{\mathbf{n}}$} & \multicolumn{2}{|c|}{$\begin{array}{l}\text { Albumin im Liquor } \\
{[\mathrm{mg} / \mathrm{l}]}\end{array}$} & \multicolumn{2}{|c|}{$\begin{array}{l}\text { Lysozym im Liquor } \\
\text { [Lysozymeinheiten } \\
\text { pro Liter] }\end{array}$} & \multicolumn{2}{|c|}{ Regression/Korrelation } \\
\hline & & $\overline{\mathbf{x}}$ & $s_{\mathbf{x}}$ & $\overline{\mathbf{y}}$ & $\mathrm{sy}_{\mathrm{y}}$ & $r$ & $\begin{array}{l}\text { signifikant } \\
\alpha=5 \%\end{array}$ \\
\hline Referenzpersonen & 21 & 158,33 & 60,18 & 16740 & 4950 & 0,403 & nein \\
\hline \multicolumn{8}{|l|}{ Patienten } \\
\hline $\begin{array}{l}\text { Bakt. Meningitis } \\
\text { Virale Meningititis } \\
\text { Multiple Sklerose } \\
\text { Chron. entzündl. Erkrankungen } \\
\text { Andere neurologische Syndrome }\end{array}$ & $\begin{array}{l}20 \\
29 \\
19 \\
28 \\
51\end{array}$ & $\begin{array}{r}1676,70 \\
593,28 \\
236,21 \\
326,25 \\
547,33\end{array}$ & $\begin{array}{r}2261,16 \\
577,76 \\
147,85 \\
242,60 \\
858,80\end{array}$ & $\begin{array}{r}150750 \\
38970 \\
38260 \\
44290 \\
44390\end{array}$ & $\begin{array}{r}132960 \\
31120 \\
30570 \\
29790 \\
31230\end{array}$ & $\begin{array}{r}-0,000 \\
0,266 \\
0,043 \\
-0,047 \\
-0,061\end{array}$ & $\begin{array}{l}\text { nein } \\
\text { nein } \\
\text { nein } \\
\text { nein } \\
\text { nein }\end{array}$ \\
\hline
\end{tabular}


Zusammenhang der Lysozymaktivität in Liquor und Serum

Abbildung 4 gibt die Wertepaare der Lysozymaktivitäten in Liquor und Serum wieder von Referenzpersonen und von Patienten. Ein Zusammenhang ließ sich optisch nicht erkennen. Die Fälle mit einer erheblichen Störung der Blut-Liquor-Schranke, kenntlich an einer hohen Albuminkonzentration im Liquor und niedriger Zellzahl, wurden besonders gekennzeichnet. Ein optischer Zusammenhang dieser Wertepaare ließ sich ebenfalls nicht erkennen.

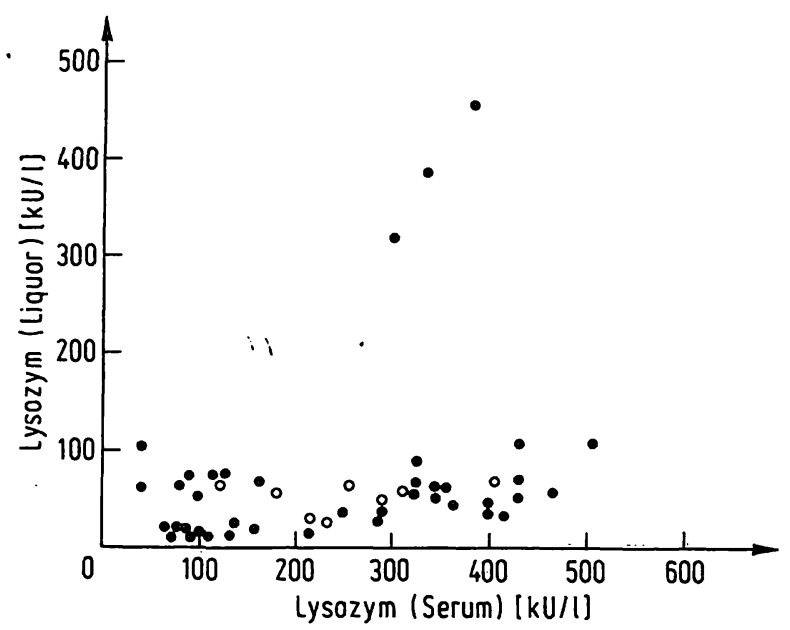

Abb. 4. Korrelation von Lysozymaktivität in Liquor und Serum. $\mathrm{O}=$ Patienten mit Störungen der Blut-Liquor-Schranke $(n=8), 0=$ Referenzpersonen $(n=6)$ und Patienten mit verschiedenen neurologischen Erkrankungen $(n=$ 34).

\section{Diskussion}

\section{Methoden}

Die Bestimmungen der Lysozymaktivität in Liquor und Serum haben hinsichtlich

- der Normbereichsgrenzen,

- der Qualität und Richtigkeit in Abhängigkeit von den zugrunde liegenden Substraten und Meßverfahren

- sowie in den Angaben der katalytischen Aktivität in Einheiten oder Gewicht pro Volumeneinheit

zu unterschiedlichen Ergebnissen geführt $(13,7,9)$.

Aus diesem Grunde wurden zwei Methoden - der

Diffusions- und Lysetest in Agargel (lysoplate-method, 16) und eine turbidimetrische Methode (17) miteinander verglichen.

Beiden Methoden gemeinsam ist die Quantifizierung der Lysozymaktivität durch Lyse von Bakterienzellwänden, in denen als Substrat Peptidoglycan enthal- ten ist (20). Die Qualitäts- und Richtigkeitskontrollen der Methoden lieferten Ergebnisse, die vergleichende Untersuchungen zuließen. Die untere Nachweisbarkeitsgrenze von 10000 Lysozymeinheiten pro Liter machte nicht in jedem Falle eine quantitative Bestimmung der Lysozymaktivität im Liquor möglich. In allen Liquorproben konnte mit dem Diffusionstest ein kleiner Lysehof um die Einfüllöffnung nachgewiesen werden. Dieser war jedoch unterhalb eines Durchmessers von $4,5 \mathrm{~mm}$ quantitativ nicht auswertbar. In den Serumproben war die $\mathrm{Be}=$ stimmung der Lysozymaktivität in jedem Fall möglich. Die Korrelation beider Meß̈methoden unter Einsatz von Serumproben ergab einen Pearson-r von 0,95 bei einer Signifikanz von $\alpha \equiv 0,1 \%$ als Hinweis auf eine gute Ubereinstimmung beider Methoden, während der Verlauf der Regressionsgeraden auf Unterschiede im Meßergebnis hinweist (Abb. 1). Bei gleichem Substrat (Micrococcus lysodeicticus) und demselben Humanlysozym-Material sowie glei= chem Puffer ergeben sich die Unterschiede in beiden Methoden in einer Zeitdifferenz (Ëinwirkzeit des Enzyms) und in einer Temperaturdifferenz:

- 1,5 Minuten (Mittelwertberechnung von $3 \mathrm{Ab}=$ sorptionsdifferenzen) bei $25^{\circ} \mathrm{C}=$ turbidimetrische Messung

- und einer Einwirkzeit vọn 24 Stunden bèi $37^{\circ} \mathrm{C}$ $\doteq$ Diffusion im Agargel.

Auf Grund der besseren Präzision und Richtigkeit des Diffusionstestes gegenübèr dem turbidimètrischen Test in der Serie und der guten Präzision und Richtigkeit von Tag zu Tag (vergleiche Ergebnisse) wurde zur Erstellung des Referenzbereichs und der Untersuchung von Liquores und Seren von Patienten der Diffusionstest herangezogen.

Eine Lysozym-Einheit des als Standard verwandten Humanlysozyms (Fa. Selfact AB., Stockholm) war definiert als Abnahme von 0,001 Absorptionseinheiten pro Minute bei $1 \mathrm{~cm}$ Schichtdicke der Küvetten bèi einer Micrococcus-Suspension in $100 \mathrm{mmol} / \mathrm{l}$ $\mathrm{KH}_{2} \mathrm{PO}_{4}$ Puffer, $\mathrm{pH}=7,0$ bei $25^{\circ} \mathrm{C}(19)$. Unter diesen $\overline{\mathrm{B} e d i n g u n g e n}$ erhielt das gefriergetrocknete $\overline{\mathrm{Hu}}=$ manlysozym der Fa. Selfact, Stockholm bei einem Reinheitsgrad von $75-85 \%$ : 40100 Lysozymeinhei-ten pro mg Trockengewicht.

Die hier zugrunde gelegten Referenzwerte wurdẹn auf Humanlysozymaktivität bezogen. Die Werte des Referenzkollektivs erstreckten sich bis 27000 Lysozymeinheiten pro Liter (Abb. 2, Tạb. 4).

\section{Referenzpersonen}

Grundlāge für die Ermittlung voñ Referenźbereichen waren Patienten, die wegen eines Verdachtes 
auf eine neurologische Erkrankung punktiert wurden. Letztere konnte ausgeschlossen werden. Damit handelte es sich im strengen Sinne nicht um ,Normalpersonen" (1).

Sämtliche klinisch-chemischen Kenngrößen lagen im Normbereich. Als Referenzbereich wurde der 95\%Vertrauensbereich herangezogen (Tab. 2, 3, 4).

\section{Patienten}

Besonders hohe Lysozymaktivitäten im Liquor wurden bei bakteriellen Meningitiden mitgeteilt. Dieses wurde als diagnostisches Kriterium für das Vorliegen einer bakteriellen Meningitis angesehen $(4,5)$. Beim Vergleich mit Kollektiven neurologischer Erkrankungen anderer Genese sind Lysozymaktivitäten oberhalb von 105000 Lysozymeinheiten pro Liter (Human-Lysozym) mit hoher Wahrscheinlichkeit einer bakteriellen Meningitis zuzuordnen (Abb. 2). Jedoch ist ein solches Ergebnis für eine bakterielle Meningitis nicht spezifisch, da auch bei andersartigen neurologischen Erkrankungen hohe Aktivitäten gemessen werden konnten (Tab. 1). Damit kann vermutet werden, daß auch andere Noxen als Bakterien die Lysozymaktivität im Liquor steigern können.

Lysozym stammt vorwiegend aus dem Stoffwechsel von Makrophagen, neutrophilen Granulocyten und deren Vorstufen direkt aus dem Knochenmark und aus Monozyten $(10,8,15)$. Hingegen weisen RESZellen und lymphoide Zellen nur eine sehr geringe Lysozymaktivität auf, die für unsere Betrachtungen kaum ins Gewicht fallen dürften (3). Lysozym, von Leukocyten, Makrophagen, Monocyten stammend, könnte demnach aus liquornahen Räumen in den $\mathrm{Li}$ quor gelangen oder im Liquor selbst gebildet werden (2). Andererseits wäre ein Úbertritt aus dem Plasma - ähnlich dem Albumin -, das ausschließlich in der Leber gebildet wird, denkbar (9).

Ein Zusammenhang der Lysozymaktivität im Liquor mit der Zellzahl (Leukocytenkonżentration) konnte gerade für bakkterielle Meningitiden nicht nachgewiesen werden, obwohl hier ein besonders hoher Zellanteil neutrophiler Granulocyten im Liquor vorliegt $(6,9$, Tab. 3). Dagegen ergab sich eine signifikante Korrelation der Wertepaare von Patienten mit viralen Meningoencephalitiden und mit multipler Sklerose (Tab. 3). Bei diesen Erkrankungen handelt es sich aber vorwiegend um eine Vermehrung lymphoider Zellen im Liquor, so daß der Zusammenhang mit der Zellzahl im Liquor nicht als unmittelbar kausal anzusehen ist. Ein Zusammenhang mit einer Lysozymproduktion in den liquornahen Räumen des ZNS durch dort angereicherte neutrophile Granulocyten und Makrophagen in den infiltrierten Herden muß vermutet werden. Eine solche An- reicherung von Lysozym-produzierenden Zellen zeigt sich aber auch bei nicht-bakteriellen Erkrankungen des Zentralnervensystems, möglicherweise als Zeichen der sekundären entzündlichen Zellreaktion, die sich dem Liquor nicht mitteilt (Tab. 3).

Da Erkrankungen des ZNS häufig mit einer Schrankenstörung zwischen Blut und Liquor einhergehen, wurde der Úbertritt von Lysozym aus dem Serum vermutet und eine Abhängigkeit der Lysozymaktivität im Liquor von der des Serums angenommen $(9$, 14). Eine Abhängigkeit der Lysozymaktivität zwischen den Kompartimenten Serum und Liquor ließ sich weder für die Gesamtzahl der untersuchten Fälle noch für die nicht-entzündlichen Erkrankungen erkennen, die mit einer Schrankenstörung zwischen Blut und Liquor einhergehen, ohne Vermehrung der Leukocytenzahl im Liquor (Abb. 4). Auch bestand keine statistische Korrelation als Hinweis auf einen gleichzeitigen Úbertritt mit Albumin aus dem Plasma (Tab. 4). Demnach verhielten sich die Lysozymaktivitäten in beiden Kompartimenten unabhängig voneinander. Dieses schließt unterschiedliche Mechanismen der Hemmung der Lysozymaktivität nicht aus (12). Eine gleichzeitige Bestimmung in $\mathrm{Li}$ quor und Serum ist aus diagnostischen Gründen nicht erforderlich.

Bei bakteriellen Meningoencephalitiden nahm der Glucosegehalt des Liquors ab, als Auswirkung eines erhöhten Verbrauchs durch den Stoffwechsel von Bakterien und Zellen (11). Die negative, statistisch signifikante Korrelation des Glucosegehaltes mit der Lysozymaktivität im.Liquor (Tab. 2) bei fehlender Korrelation mit dem Zellgehalt im Liquor selbst (Tab. 3) spricht für den Zusammenhang mit dem Zellstoffwechsel in den liquornahen Räumen. Das Lysozym könnte demnach aus Zellen stammen, die in liquornahen Räumen an der bakteriell entzündlichen Reaktion beteiligt sind.

Die Lysozymaktivität des Liquors sank während der Behandlung einer bakteriellen Meningoencephalitis mit steigender Antibiotikakonzentration schneller ab, als die Glucosekonzentration des Liquors anstieg (Abb. 3). Die Vermutung liegt nahe, daß die Lysozymaktivität im Liquor ursächlich durch das Antibiotikum beeinflußt wurde infolge der bakteriziden bzw. bakteriostatischen Wirkung und so eine Kenngröße für die Wirksamkeit der Antibiotikatherapie sein könnte. Ähnliche Beobachtungen teilte Gekle et al. (5) mit bei der Untersuchung von Patientenkollektiven mit bakterieller Meningitis. Auch unter Azathioprintherapie nach Nierentransplantation wurde eine im Zusammenhang mit der Behandlung sinkende Lysozymkonzentration im Serum mitgeteilt (10). 


\section{Literatur}

1. Büttner, H. (1973) In: Optimierung der Diagnostik (Lang, H., Rick, W. \& Roka, L., eds.) Springer Berlin, Heidelberg, New York, pp. 233-241.

2. Dick, W., Braun, O. H., Nagel, W. \& Theilmann, L. (1980) Monatsschr. Kinderheilk. 128, 472-475.

3. Finch, S. C., Castro, O., Lippman, M. E., Donadioy, I. A. \& Perillie, P. E. (1974) In: Lysozyme (Osserman, E. F., Canfield, R. E. \& Beychok, Sh., eds.) Academic Press, New York, London, pp. 335-345.

4. Gekle, D., Kult, J. \& Roth, R. (1977) Klin. Wochenschr. 55, 189-191.

5. Gekle, D., Kult, J. \& Roth, R. (1980) Lab. Med. 4, 71-74.

6. Grossgebauer, K., Pohle, H. D. \& Langmaack, H. (1968) Klin. Wochenschr. 46, 1127.

7. Hansen, N. E., Karle, H., Andersen, V. \& Đlgaard, K. (1972) J. Clin. Invest. 51, 1146-1155.

8. Hansen, N. E. \& Andersen, V. (1973) Brit. J. Haematology $24,613$.

9. Hansen, N. E., Karle, H., Jensen, A. \& Bock, E. (1977) Acta Neurol. Scand. 55, 418-424.

10. Horpacsy, G., Zinsmeyer, J., Schröder, K. \& Mebel, M. (1978) Clin. Chem. 24, 74-79.

11. Kauerz, U., Weisner, B., Bernhardt, W. \& Rehpennining, W. (1982) Nervenarzt 53, 91-97.
12. Kauerz, U. \& Weisner, B. (1981) In: Berichte der OGKG, III. International Congress of Clinical Enżymology, Salzburg, Sept. 6-9, Jahrg. 4, Heft 3, p. 133.

13. Kleine, T. O. (1979) In: Bericht über die Kleinkonferreṇż der Deutschen Ges. f. Klin. Chemie am 13. und 14. Oktober 1978 in Marburg/Lahn, (1978) J. Clin. Chem. Clin. Biochem. 17, 506.

14. Kleine, T. O. (1980) In: Neue Labormethoden für die Liquordiagnostik (Kleine, T. O. Hrsg.) Thieme Verlag Stuttgart, New York, pp. 59-69.

15. Ohta, H. \& Osserman, E. F. (1972) Tohoku J. Exp. Med. 107, 229.

16. Osserman, E. F. \& Lawlor, D. P. (1966) J. Exp̄. Med. 124, 921-952.

17. Priokop, D. J. \& Davidson, W. D. (1964) New Eṇgl. J. Med. $270,269-274$.

18. Sachs, L. (1969) Statistische Auswertungsmethoden, Sprin= ger Berlin, Heidelberg, New York, pp. $80=81$.

19. Shugar; D. (1952) Biochim. Biophys. Acta $8,302-309$.

20. Strominger, J. L. \& Tripper, D. J. (1974) In: Lysozyme, (Osserman; E. F., Canfield, R. \& Beychok, S., eds.); Academic Press, Neww York, London, pp. 169-184.

21. Weisner, B. \& Kauerz, U. (1979) Medizintechnik 3, 89-92.

PD Dr. B. Weisner

Neurologische Universitätsklinik

und Poliklinik

Martinistr. 52

D-2000 Hamburg 20 